\title{
Prognostic Value of Secretoneurin in Critically III Patients With Infections
}

\author{
Helge Røsjø, MD, $\mathrm{PhD}^{1,2}$; Mats Stridsberg, MD, PhD³; Anett H. Ottesen, MSc ${ }^{1,2,4}$; Ståle Nygård, $\mathrm{PhD}^{2,5}$; \\ Geir Christensen, MD, PhD, MHA ${ }^{2,4}$; Ville Pettilä, MD, $\mathrm{PhD}^{6,7}$; Rita Linko, MD, $\mathrm{PhD}^{6}$; \\ Sari Karlsson, $\mathrm{MD}, \mathrm{PhD}^{8}$; Tero Varpula, $\mathrm{MD}, \mathrm{PhD}^{6}$; Esko Ruokonen, $\mathrm{MD}, \mathrm{PhD}^{9}$; \\ Torbjørn Omland, MD, PhD, MPH ${ }^{1,2}$; on behalf of the FINNSEPSIS and FINNALI Study Groups
}

\begin{abstract}
Objectives : Secretoneurin is produced in neuroendocrine cells, and the myocardium and circulating secretoneurin levels provide incremental prognostic information to established risk indices in cardiovascular disease. As myocardial dysfunction contributes to poor outcome in critically ill patients, we wanted to assess the
\end{abstract}

1'Division of Medicine, Akershus University Hospital, Lørenskog, Norway.

${ }^{2}$ Center for Heart Failure Research, Institute of Clinical Medicine, University of Oslo, Oslo, Norway.

${ }^{3}$ Department of Medical Sciences, Uppsala University, Uppsala, Sweden. ${ }^{4}$ Institute for Experimental Medical Research, Oslo University Hospital, Oslo, Norway.

${ }^{5}$ Bioinformatics core facility, University of Oslo and Oslo University Hospital, Oslo, Norway.

${ }^{6}$ Department of Intensive Care Medicine, University of Helsinki and Helsinki University Hospital, Helsinki, Finland.

${ }^{7}$ Department of Intensive Care Medicine, Bern University Hospital, University of Bern, Bern, Switzerland.

${ }^{8}$ Department of Intensive Care Medicine, Tampere University Hospital, Tampere, Finland.

Intensive Care Medicine, Kuopio University Hospital, Kuopio, Finland.

Supplemental digital content is available for this article. Direct URL citations appear in the printed text and are provided in the HTML and PDF versions of this article on the journal's website (http://journals.lww.com/ ccmjournal).

Supported, in part, by Akershus University Hospital. The sponsor had no role in any of the following: design and conduct of the study, collection, management, analysis and interpretation of the data, or preparation, review and approval of the article.

A complete list of the investigators who participated in the FINNSEPSIS and FINNALI studies is presented in Appendix 1 (Supplemental Digital Content 1, http://links.Iww.com/CCM/B846).

Drs. Røsjø, Stridsberg, Christensen, and Omland are partners in a patent filed by the University of Oslo regarding the use of secretoneurin as a biomarker in patients with cardiovascular disease and patients with critical illness. They have received funding relating to this work from Akershus University Hospital. Drs. Stridsberg, Christensen, and Omland have ownership interest in Cardinor AS that aims to promote secretoneurin as a biomarker. The remaining authors have disclosed that they do not have any potential conflicts of interest.

For information regarding this article, E-mail: helge.rosjo@medisin.uio.no Copyright (C) 2016 by the Society of Critical Care Medicine and Wolters Kluwer Health, Inc. All Rights Reserved.

DOI: 10.1097/CCM.0000000000001832 prognostic value of secretoneurin in two cohorts of critically ill patients with infections.

Design: Two prospective, observational studies.

Setting: Twenty-four and twenty-five ICUs in Finland.

Patients: A total of 232 patients with severe sepsis (cohort \#1) and 94 patients with infections and respiratory failure (cohort \#2). Interventions: None.

Measurements and Main Results: We measured secretoneurin levels by radioimmunoassay in samples obtained early after ICU admission and compared secretoneurin with other risk indices. In patients with severe sepsis, admission secretoneurin levels (logarithmically transformed) were associated with hospital mortality (odds ratio, $3.17[95 \% \mathrm{Cl}, 1.12-9.00] ; p=0.030$ ) and shock during the hospitalization (odds ratio, 2.17 [1.06-4.46]; $p=0.034$ ) in analyses that adjusted for other risk factors available on ICU admission. Adding secretoneurin levels to age, which was also associated with hospital mortality in the multivariate model, improved the risk prediction as assessed by the category-free net reclassification index: 0.35 $(95 \% \mathrm{Cl}, 0.06-0.64)(p=0.02)$. In contrast, N-terminal pro-B-type natriuretic peptide levels were not associated with mortality in the multivariate model that included secretoneurin measurements, and $\mathrm{N}$-terminal pro-B-type natriuretic peptide did not improve patient classification on top of age. Secretoneurin levels were also associated with hospital mortality after adjusting for other risk factors and improved patient classification in cohort \#2. In both cohorts, the optimal cutoff for secretoneurin levels at ICU admission to predict hospital mortality was $\approx 175 \mathrm{pmol} / \mathrm{L}$, and higher levels were associated with mortality also when adjusting for Simplified Acute Physiology Score II and Sequential Organ Failure Assessment scores.

Conclusions: Secretoneurin levels provide incremental information to established risk indices for the prediction of mortality and shock in critically ill patients with severe infections. (Crit Care Med 2016; XX:00-00)

Key Words: critical illness; prognosis; secretoneurin; sepsis

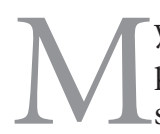
yocardial dysfunction contributes to mortality in patients with critical illness, including in patients with severe sepsis (1). However, as established risk models 
in critical illness require 24 hours of data collection $(2,3)$, currently, no test is available to identify high-risk patients at ICU admission. Cardiovascular biomarkers could prove useful for early risk assessment in critically ill patients with infections, but the established biomarkers N-terminal pro-B-type natriuretic peptide (NT-proBNP) and high-sensitivity troponin T (hs-TnT) seem to lack the desired prognostic accuracy when measured at ICU admission $(4,5)$. Accordingly, novel cardiovascular biomarkers are needed to improve the risk assessment on ICU admission.

Secretoneurin is a 33-amino acid peptide derived from the proprotein secretogranin II, which is produced in the myocardium and in neuroendocrine cells $(6,7)$. Circulating secretoneurin levels increase during profound systemic and myocardial stress $(7,8)$, and secretoneurin levels are also increased by renal failure (9). The potential of secretoneurin to integrate information from the cardiovascular, renal, and neuroendocrine system may explain the potent prognostic information recently demonstrated for secretoneurin in patients with cardiovascular disease (CVD) (9). In patients with acute heart failure and after ventricular arrhythmia-induced cardiac arrest, secretoneurin provided additional prognostic information to clinical variables and norepinephrine, NT-proBNP, and hs-TnT levels. Other groups have also found secretoneurin to predict outcome after cardiac arrest (10). In contrast, admission secretoneurin levels were not associated with outcome in patients with acute exacerbations of chronic obstructive pulmonary disease (9), although these patients had comparable severity of dyspnea and mortality rates as the acute heart failure patients. Hence, secretoneurin seems to provide incremental prognostic information to established risk indices in patients with CVD, although not being an unspecific biomarker of disease severity per se. This model is supported by the direct inhibitory effect by secretoneurin on diastolic $\mathrm{Ca}^{2+}$ leak via $\mathrm{Ca}^{2+} /$ calmodulin-dependent protein kinase II $\delta$ inhibition (9) and reduced cardiomyocyte apoptosis and myocardial ischemia/reperfusion injury by secretoneurin

\section{Derivation cohort: FINNSEPSIS Study}

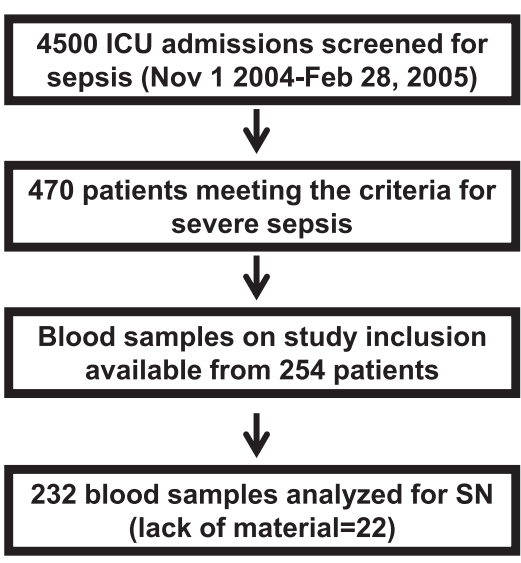

in experimental models $(11,12)$. Accordingly, as cardiovascular, renal, and neuroendocrine system dysfunctions influence outcome in patients with severe infections (1), we hypothesized that secretoneurin would provide important prognostic information in critically ill patients with infections.

\section{MATERIALS AND METHODS}

\section{Study Design}

We included patients from the two large Finnish multicenter studies FINNish SEPSIS (FINNSEPSIS)-study (cohort \#1) and the FINNish Acute Lung Injury (FINNALI)-study (cohort \#2). The FINNSEPSIS study comprised 24 ICUs, and during a 4-month period (November 1, 2004, to February 28, 2005), all adult patients ( $\geq 18 \mathrm{yr}$ ) admitted to the participating ICUs were screened daily for the American College of Chest Physicians/Society of Critical Care Medicine criteria of severe sepsis and septic shock: 1) known or suspected infection, 2) two or more criteria for systemic inflammatory response syndrome, and 3) at least one new, sepsis-induced organ dysfunction (Fig. 1) (13). Four hundred seventy patients were included, and blood samples were available from 232 patients at ICU admission (median, 9 [Q1-Q3, 3-17] hr from study inclusion to sampling) and from 201 patients after 72 hours.

Cohort \#2 was from the FINNALI study. During an 8-week period (April 16 to June 10, 2007), all patients treated with respiratory support more than 6 hours in 25 Finnish ICUs were included (14), and of the 602 patients with informed consent for and available blood samples at ICU admission (median, 6 [6-8] hr from study inclusion to sampling), 94 patients were diagnosed with pneumonia $(n=64)$ or sepsis $(n=30)$ by the treating physician at ICU admission (Acute Physiology and Chronic Health Evaluation III diagnosis group) and included in the current study with follow-up samples available in 78 patients (Fig. 1). Blood samples were stored at $-80^{\circ} \mathrm{C}$, and secretoneurin measurements were performed as retrospective post hoc analyses with measurements finalized on August 25, 2010.

The primary endpoint of this study was hospital mortality and the secondary endpoint was septic shock, which were both reported in electronic clinical report forms by the treating physician at the time of death or hospital discharge. We recorded information on comorbidities and standard clinical and laboratory variables available less than 24 hours from patient chart records in both studies. The history of CVD was defined as previous coronary artery disease or heart failure, and this was assessed
Fig. 1. Flowchart of the study. APACHE = Acute Physiology and Chronic Health Evaluation, FINNALI = FINNish Acute Lung Injury, FINNSEPSIS = FINNish SEPSIS, SN = secretoneurin.
94 patients diagnosed with pneumonia or sepsis (APACHE III diagnosis group) 
by the treating physician. The body mass index was calculated by weight $(\mathrm{kg}) /(\text { height }[\mathrm{m}])^{2}$. The Simplified Acute Physiology Score (SAPS) II and the Sequential Organ Failure Assessment (SOFA) score were calculated after 24 hours, and we also collected information on the use of inotropes during the ICU stay.

Both studies were conducted according to the Declaration of Helsinki and approved by the local Ethics Committees. Informed consent was obtained from the patient or a close relative before study commencement.

\section{Biochemical Analyses}

Secretoneurin was measured by an in-house radioimmunoassay as previously reported (7-9). The limit of detection for the secretoneurin assay is $50 \mathrm{pmol} / \mathrm{L}$, and the assay has a coefficient of variation of $9 \%$ in the lower range $(110 \mathrm{pmol} / \mathrm{L})$ and $4 \%$ in the upper range $(380 \mathrm{pmol} / \mathrm{L})$. To assess the stability of secretoneurin during long-term storage at $-80^{\circ} \mathrm{C}$, we also reanalyzed samples from another cohort (7) that had been stored for almost 7.5 years and found secretoneurin levels to be comparable with the measurements performed in 2008 (Supplementary Fig. 1, Supplemental Digital Content 2, http://links. lww.com/CCM/B847). We measured NT-proBNP by the Elecsys proBNP assay (Roche Diagnostics, Penzberg, Germany) (4) and chromogranin A ( $\mathrm{CgA})$ by a commercially available radioimmunoassay (EuroDiagnostica, Malmö, Sweden) (15). hs-TnT levels were measured by the Elecsys TNT hs STAT assay (Roche Diagnostics) in a subgroup of the patients with severe sepsis $(n=207)$ as previously reported (5). Cortisol was measured by an immunoassay in the patients who had not received corticosteroids prior to blood sampling, and free cortisol was calculated by the Coolens method (16). We calculated creatinine clearance by the Cockgroft-Gault formula (17).

\section{Statistical Analysis}

We present data as median (quartile [Q], 1-3) or absolute numbers and percentages. Biomarkers and other continuous variables deviated from the normal distribution as assessed by the Kolmogorov-Smirnov test and group differences were assessed by the Mann-Whitney $U$ test or the chi-square test, and serial measurements were tested by the related-sample Wilcoxon signed rank test. Spearman rank correlation was used to examine correlations between variables. The ability of secretoneurin to predict mortality was explored by univariate and multivariate logistic regression analyses with odds ratios (ORs) and $95 \%$ CIs. Variables associated with mortality in univariate analysis were included in multivariate models of both cohorts (backward selection). We also performed additional bivariate logistic regression analyses with secretoneurin and CVD and secretoneurin and NT-proBNP. We assessed the association between secretoneurin and septic shock during the hospitalization by univariate and multivariate logistic regression. Secretoneurin and other variables with a right-skewed distribution were transformed by the natural logarithm prior to regression analyses. Prognostic accuracy was assessed by receiver operating characteristics (ROC) curve analysis with area under the curve (AUC) presented with 95\% CI using a logistic model with hospital mortality as the response variable. The AUCs are from nested models and are presented accordingly (18). We also used ROC statistics and the Youden index J to identify the optimal cutoff points of secretoneurin. The basic risk model was built based on the independent variables of the multivariate regression analysis, which is analogous to the strategy used in previous publications by our group $(9,15)$, and we examined calibration by calculating the category-free net reclassification index (NRI) (19). We also tested the optimal secretoneurin cutoff from the FINNSEPSIS study in patients with sepsis in cohort \#2 by producing Kaplan-Meier plots and comparing the groups by the log-rank test. A $p$ value less than 0.05 was considered statistically significant. Statistical analyses were performed with SPSS for Windows version 19.0 (SPSS, Chicago, IL), MedCalc for Windows, version 12.1.4.0 (MedCalc Software, Mariakerke, Belgium), and the statistical programming language $\mathrm{R}$ (R Development Core Team, Vienna, Austria).

\section{RESULTS}

\section{Secretoneurin Levels in Patients With Severe Sepsis (Cohort \#1; FINNSEPSIS Study)}

The prevalence of common risk factors in critically ill patients was higher among hospital nonsurvivors compared with survivors (Supplementary Table 1, Supplemental Digital Content 2, http://links.lww.com/CCM/B847). The median secretoneurin level on study inclusion in patients with severe sepsis was 170 pmol/L (Q1-Q3, 131-240 pmol/L). Patients with secretoneurin levels over the median at ICU admission were more often women, had worse renal function, had higher lactate and free cortisol levels, had lower platelet count, had higher levels of admission cardiovascular biomarkers, had higher SAPS II and SOFA scores after 24 hours, and were more likely to need infusion with dobutamine and epinephrine (Table 1). Secretoneurin levels at ICU admission were positively correlated with admission NT-proBNP, hs-TnT, CgA, cortisol and lactate levels, and SAPS II and SOFA scores after 24 hours and inversely correlated with estimated creatinine clearance (Supplementary Table 2, Supplemental Digital Content 2, http://links.lww.com/CCM/B847).

\section{Predictors of Hospital Mortality in Severe Sepsis}

Hospital mortality in the patients with severe sepsis was $28 \%$ (65/232). High secretoneurin levels at ICU admission were associated with increased risk of hospital mortality: OR, 3.60 (95\% CI, 1.74-7.44) ( $p=0.001)$ (Table 2). This association was significant also after adjustment for other risk variables, including history of CVD and cardiovascular biomarkers: OR, 3.17 $(1.12-9.00)(p=0.030)$. In contrast, the associations between hospital mortality and NT-proBNP, hs-TnT, and CgA levels were not significant in multivariate analysis that also included secretoneurin. Adjusting for CVD and NT-proBNP in separate bivariate analyses did not change the association between secretoneurin and hospital mortality (Supplementary Table 3, Supplemental Digital Content 2, http://links.lww.com/CCM/ B847). The AUC of secretoneurin to predict hospital mortality in patients with severe sepsis was 0.65 (95\% CI, 0.57-0.73), and 


\section{TABLE 1. Characteristics of the Patients in the Two Cohorts as Divided by the Median} Secretoneurin Level at Admission (pmol/L)

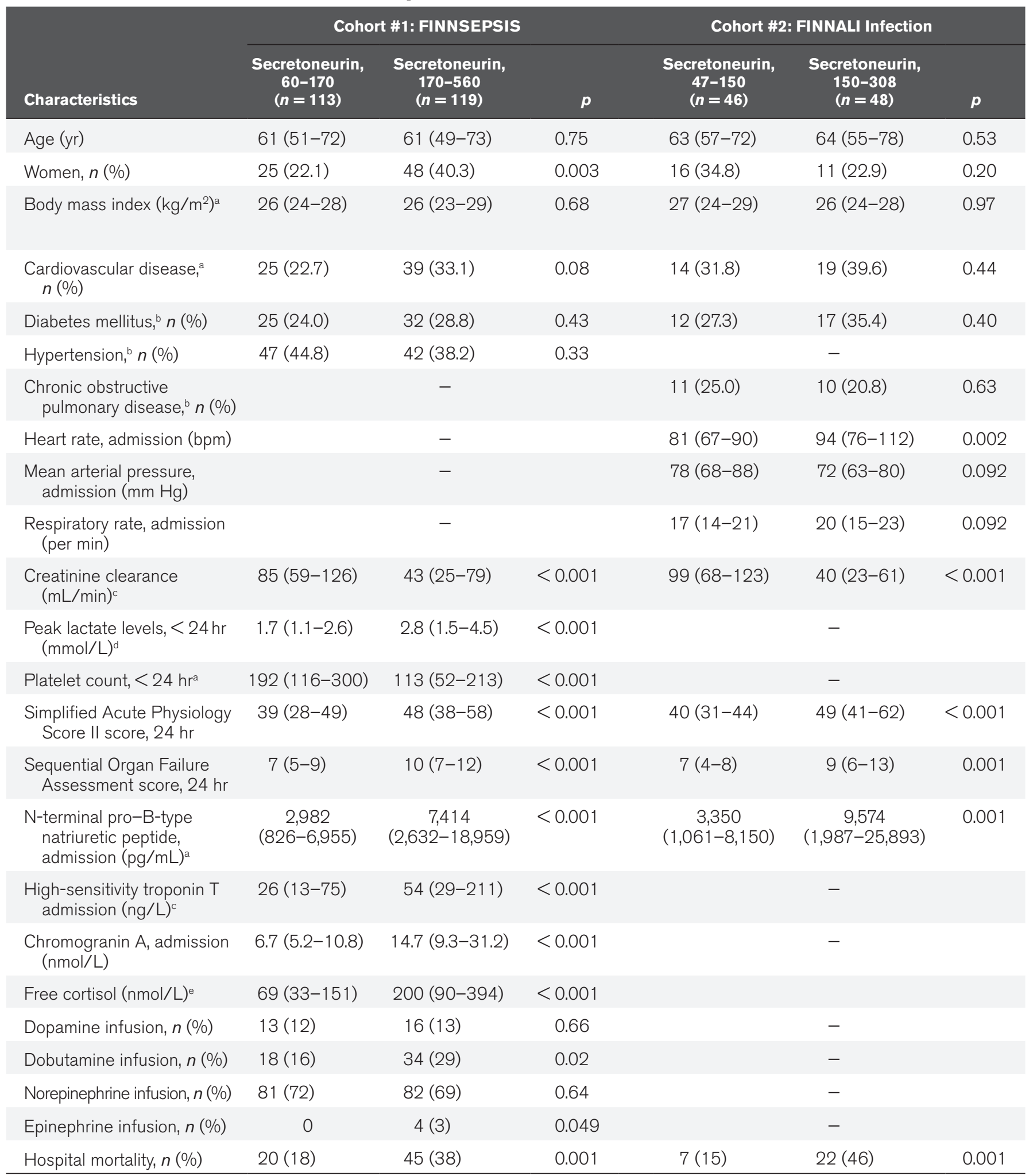

FINNALI = FINNish Acute Lung Injury, FINNSEPSIS = FINNish SEPSIS

${ }^{2}<5 \%$ missing.

${ }^{b}<10 \%$ missing.

$c<15 \%$ missing.

${ }^{\mathrm{d}}<20 \%$ missing.

${ }^{\mathrm{e}}$ Available in 133 patients. 


\section{TABLE 2. Associations Between Variables Available on Study Inclusion and Hospital} Mortality in the Two Cohorts

\begin{tabular}{|c|c|c|c|c|c|c|}
\hline \multirow[b]{2}{*}{ Characteristics } & \multicolumn{3}{|c|}{ Cohort \#1: FINNSEPSIS } & \multicolumn{3}{|c|}{ Cohort \#2: FINNALI Infection } \\
\hline & OR & $95 \% \mathrm{Cl}$ & $p$ & OR & $95 \% \mathrm{Cl}$ & p \\
\hline \multicolumn{7}{|l|}{ Univariate analysis } \\
\hline Age, per 1-yr increase & 1.05 & $1.02-1.07$ & $<0.001$ & 1.03 & $0.99-1.06$ & 0.13 \\
\hline Gender (female vs male) & 1.70 & $0.93-3.10$ & 0.08 & 0.92 & $0.35-2.44$ & 0.87 \\
\hline Body mass index, per 1 -unit increase & 1.00 & $0.93-1.06$ & 0.87 & 0.97 & $0.90-1.05$ & 0.48 \\
\hline Cardiovascular disease & 2.51 & $1.35-4.64$ & 0.004 & 1.54 & $0.62-3.82$ & 0.36 \\
\hline Diabetes mellitus & 2.56 & $1.34-4.88$ & 0.004 & 1.32 & $0.51-3.37$ & 0.57 \\
\hline Hypertension & 1.13 & $0.47-1.73$ & 0.76 & & - & \\
\hline Chronic obstructive pulmonary disease & & - & & 0.89 & $0.31-2.60$ & 0.83 \\
\hline Heart rate on inclusion (bpm) & & - & & 1.00 & $0.98-1.02$ & 0.96 \\
\hline Mean arterial pressure on inclusion $(\mathrm{mm} \mathrm{Hg})$ & & - & & 0.99 & $0.96-1.02$ & 0.35 \\
\hline Respiratory rate on inclusion (per min) & & - & & 1.01 & $0.94-1.08$ & 0.81 \\
\hline Positive blood culture & 0.90 & $0.47-1.73$ & 0.76 & & - & \\
\hline Proton pump inhibitor use & 1.50 & $0.65-3.47$ & 0.35 & & - & \\
\hline Creatinine clearance $(\mathrm{mL} / \mathrm{min})$ & 0.45 & $0.29-0.71$ & $<0.001$ & 0.36 & $0.17-0.78$ & 0.009 \\
\hline Platelet count $\left(\times 10^{9} / \mathrm{L}\right)$ & 0.82 & $0.60-1.14$ & 0.24 & & - & \\
\hline Peak lactate $<24 \mathrm{hr}(\mathrm{mmol} / \mathrm{L})$ & 1.16 & $1.03-1.31$ & 0.012 & & - & \\
\hline Total fluid resuscitation volume $<24 \mathrm{hr}(\mathrm{L})$ & 1.05 & $0.98-1.14$ & 0.19 & 1.09 & $0.91-1.32$ & 0.36 \\
\hline Steroid infusion & 1.14 & $0.64-2.02$ & 0.66 & & - & \\
\hline $\mathrm{N}$-terminal pro-B-type natriuretic peptide $(\mathrm{pg} / \mathrm{mL})$ & 1.32 & $1.08-1.62$ & 0.006 & 1.62 & $1.16-2.28$ & 0.005 \\
\hline High-sensitivity troponin $T$ (ng/L) & 1.28 & $1.03-1.59$ & 0.026 & & - & \\
\hline Chromogranin A (nmol/L) & 1.87 & $1.30-2.68$ & 0.001 & & - & \\
\hline Secretoneurin (pmol/L) & 3.60 & $1.74-7.44$ & 0.001 & 16.21 & $3.43-76.53$ & $<0.001$ \\
\hline $\begin{array}{l}\text { Sepsis (Acute Physiology and Chronic Health } \\
\text { Evaluation diagnosis) }\end{array}$ & & - & & 2.29 & $0.92-5.74$ & 0.08 \\
\hline \multicolumn{7}{|l|}{ Multivariate analysis (variables retained in the final model) ${ }^{a}$} \\
\hline Secretoneurin (pmol/L) & 3.17 & $1.12-9.00$ & 0.030 & 11.60 & $1.37-98.32$ & 0.025 \\
\hline Age & 1.03 & $1.01-1.07$ & 0.038 & 1.05 & $1.001-1.11$ & 0.044 \\
\hline
\end{tabular}

FINNALI $=$ FINNish Acute Lung Injury, FINNSEPSIS $=$ FINNish SEPSIS, OR $=$ odds ratio.

${ }^{a}$ All variables available on study inclusion and associated with the outcome in univariate analyses in one of the cohorts were included in the multivariate models, that is age, history of cardiovascular disease and diabetes mellitus, lactate, creatinine clearance, and cardiovascular biomarkers.

the AUC of NT-proBNP was 0.63 (0.54-0.71) (Supplementary Fig. 2, Supplemental Digital Content 2, http://links.lww.com/ $\mathrm{CCM} / \mathrm{B} 847)$. Adding secretoneurin to age increased the AUC from $0.66(0.59-0.74)$ to $0.71(0.64-0.78)$. In contrast, the addition of NT-proBNP to age changed the AUC from 0.66 (0.59$0.74)$ to $0.68(0.60-0.75)$. Secretoneurin at ICU admission also improved the classification of patients on top of age as assessed by category-free NRI: 0.35 (95\% CI, 0.06-0.64) ( $p=0.02$ ). The effect by secretoneurin was primarily to downgrade risk in patients considered of high risk but who did not experience an event during the index hospitalization (Supplementary Fig.
3, Supplemental Digital Content 2, http://links.lww.com/CCM/ B847). In contrast, NT-proBNP did not provide a significant improvement in NRI: $-0.03(-0.33$ to 0.26$)(p=0.82)$ (Supplementary Fig. 4, Supplemental Digital Content 2, http://links. lww.com/CCM/B847). The optimal secretoneurin concentration to predict hospital mortality was $175 \mathrm{pmol} / \mathrm{L}$ with sensitivity $68 \%$ and specificity $60 \%$. Patients with secretoneurin levels over $175 \mathrm{pmol} / \mathrm{L}$ at ICU admission had increased risk of hospital mortality (Fig. 2), also after adjustment for SAPS II score (OR, $2.08[1.08-3.99] ; p=0.028)$ and SOFA score (OR, 2.15 $[1.12-4.11] ; p=0.022)$. 


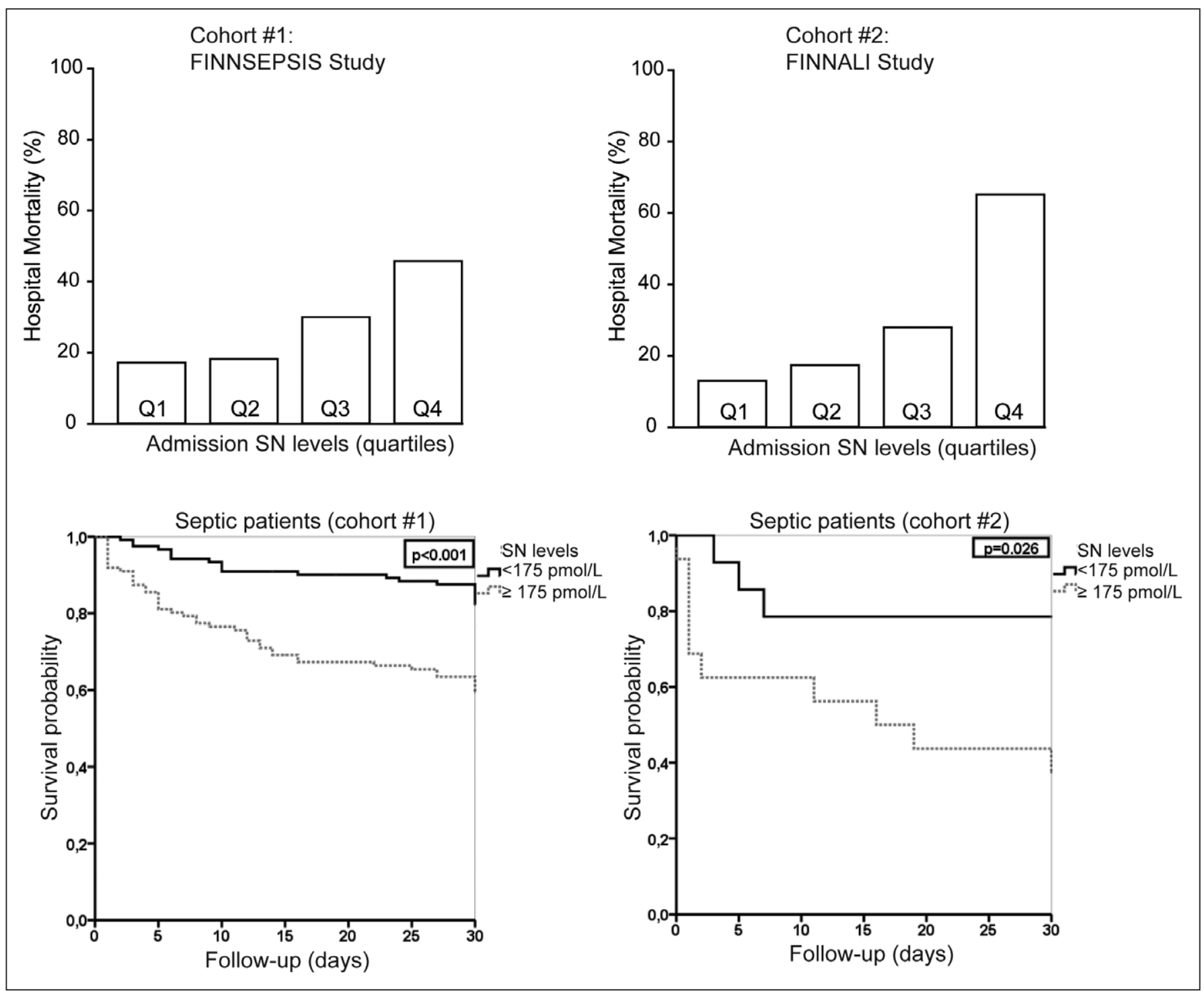

Fig. 2. Mortality according to quartiles of secretoneurin (SN) measured on inclusion in the two cohorts. Mortality for septic patients in cohort \#1 and \#2 stratified according to the cutoff of SN $175 \mathrm{pmol} / \mathrm{L}$ is also presented, and the groups were compared by the log-rank test. FINNALI = FINNish Acute Lung Injury, FINNSEPSIS = FINNish SEPSIS.

\section{Secretoneurin Levels and Septic Shock in Patients With Severe Sepsis}

Of the 232 patients with severe sepsis, 135 patients (58\%) were diagnosed with shock during the ICU stay. Increasing age and high NT-proBNP, hs-TnT, and secretoneurin levels at ICU admission were associated with shock in univariate analyses, but only secretoneurin levels and age were associated with septic shock in the multivariate analysis (Table 3). The ROCAUC of secretoneurin to predict hospital mortality in patients with septic shock during the hospitalization was 0.63 (95\% CI, $0.55-0.71)$, and the ROC-AUC to predict mortality in patients without shock was 0.65 (0.54-0.74).

\section{Secretoneurin Levels and Hospital Mortality in Cohort \#2 (FINNALI Study)}

Hospital mortality in cohort \#2 was 31\% (29/94), and the median secretoneurin level on study inclusion was $150 \mathrm{pmol} / \mathrm{L}$
(Q1-Q3, 108-192 pmol/L). Patient characteristics according to mortality in cohort \#2 are presented in Supplementary Table 4 (Supplemental Digital Content 2, http://links.lww.com/CCM/ B847). Patients with secretoneurin levels over the median at ICU admission had worse renal function and higher NTproBNP levels and SOFA and SAPS II scores compared with the other patients (Table 1). Secretoneurin levels in this cohort were also positively correlated with NT-proBNP levels and SAPS II and SOFA scores and inversely correlated with estimated creatinine clearance (Supplementary Table 5, Supplemental Digital Content 2, http://links.lww.com/CCM/B847).

Including the same variables into the multivariate model as used in cohort \#1, secretoneurin levels and age were the only two variables independently associated with hospital mortality (Table 2). Adjusting for CVD and NT-proBNP in separate bivariate analyses did not change the association between secretoneurin and hospital mortality (Supplementary Table 3, 


\section{TABLE 3. Association Between Variables Available on Inclusion in the Study and Septic Shock During the ICU Stay in the FINNish SEPSIS Study (Cohort \#1)}

\begin{tabular}{|c|c|c|c|}
\hline Characteristics & OR & $95 \% \mathrm{Cl}$ & $p$ \\
\hline \multicolumn{4}{|l|}{ Univariate analysis } \\
\hline Age, per 1-yr increase & 1.03 & $1.01-1.04$ & 0.007 \\
\hline Gender (female vs male) & 0.96 & $0.55-1.69$ & 0.89 \\
\hline Body mass index, per 1-unit increase & 1.03 & $0.97-1.09$ & 0.39 \\
\hline Cardiovascular disease & 1.68 & $0.92-3.09$ & 0.09 \\
\hline Diabetes mellitus & 1.07 & $0.58-1.97$ & 0.84 \\
\hline Hypertension & 1.12 & $0.65-1.95$ & 0.68 \\
\hline Creatinine clearance $(\mathrm{mL} / \mathrm{min})$ & 0.76 & $0.51-1.13$ & 0.18 \\
\hline Lactate $(\mathrm{mmol} / \mathrm{L})$ & 1.06 & $0.95-1.20$ & 0.30 \\
\hline Platelet count $\left(\times 10^{9} / \mathrm{L}\right)$ & 0.86 & $0.63-1.16$ & 0.31 \\
\hline $\mathrm{N}$-terminal pro-B-type peptide $(\mathrm{pg} / \mathrm{mL})$ & 1.22 & $1.02-1.44$ & 0.026 \\
\hline High-sensitivity troponin $T(n g / L)$ & 1.26 & $1.03-1.54$ & 0.022 \\
\hline Chromogranin A (nmol/L) & 1.39 & $0.99-1.95$ & 0.059 \\
\hline Secretoneurin (pmol/L) & 2.13 & $1.11-4.08$ & 0.023 \\
\hline \multicolumn{4}{|c|}{ Multivariate analysis: final model (variables retained in the final model) } \\
\hline Age, yr & 1.02 & $1.01-1.04$ & 0.020 \\
\hline Secretoneurin (pmol/L) & 2.17 & $1.06-4.46$ & 0.034 \\
\hline
\end{tabular}

$\mathrm{OR}=$ odds ratio.

Supplemental Digital Content 2, http://links.lww.com/CCM/ B847). The AUC of secretoneurin to predict hospital mortality in cohort \#2 was 0.74 (95\% CI, 0.64-0.85), and the AUC of NT-proBNP was $0.69(0.58-0.81)$ (Supplementary Fig. 2, Supplemental Digital Content 2, http://links.lww.com/CCM/ B847). Adding secretoneurin to age increased the AUC from $0.61(0.48-0.74)$ to $0.76(0.66-0.86)$, whereas the addition of NT-proBNP to age changed the AUC from $0.61(0.48-0.74)$ to $0.68(0.56-0.80)$. Secretoneurin improved the classification of patients on top of age as assessed by category-free NRI (0.70 [95\% CI, 0.28-1.11]; $p<0.001)$ with secretoneurin both upgrading risk in patients with subsequent events and downgrading risk in patients with no event during the hospitalization (Supplementary Fig. 5, Supplemental Digital Content 2, http://links.lww.com/CCM/B847). We identified a secretoneurin concentration of $170 \mathrm{pmol} / \mathrm{L}$ as the optimal cutoff to predict hospital mortality in cohort \#2, and patients with secretoneurin levels over $170 \mathrm{pmol} / \mathrm{L}$ had increased risk of mortality also after adjustment for SAPS II and SOFA scores, which both were calculated after 24 hours: OR, 6.96 (2.60-18.59) $(p<0.001)$ for both. Assessing only the patients of cohort \#2 with sepsis $(n=30)$, the AUC of secretoneurin to predict hospital mortality $(n=13 ; 43 \%)$ was $0.72(0.52-0.86)$. The cutoff of secretoneurin $175 \mathrm{pmol} / \mathrm{L}$ derived from cohort \#1 was associated with mortality in the septic patients of cohort \#2 (Fig. 2) with sensitivity $70 \%$ and specificity $65 \%$. There were no interactions between the time of blood sampling and secretoneurin levels for the endpoint hospital mortality and septic shock in cohort \#1 or \#2.

\section{Changes in Secretoneurin Levels During ICU Admission}

We found that secretoneurin levels decreased significantly from study inclusion to the second timepoint for blood sampling in both cohorts (Supplementary Fig. 6, Supplemental Digital Content 2, http://links.lww.com/CCM/B847). There were close correlations between secretoneurin levels measured on study inclusion and at later timepoints: $\rho=0.65$ (cohort $\# 1)$ and $\rho=0.74$ (cohort \#2) $(p<0.001)$ for both. Secretoneurin levels after 72 hours in cohort \#1 separated hospital survivors from nonsurvivors (median, 170 [Q1-Q3, 140-240] vs 150 [120-173] pmol/L; $p=0.002$, whereas secretoneurin levels after 48 hours in cohort \#2 did not separate between survivors and nonsurvivors (129 [106-152] vs 118 [99-148] $\mathrm{pmol} / \mathrm{L} ; p=0.33)$. The AUC of secretoneurin after 72 hours in cohort \#1 for hospital mortality was 0.65 (95\% CI, 0.58-0.71), whereas the AUC of secretoneurin after 48 hours in cohort \#2 for hospital mortality was $0.57(0.45-0.68)$.

\section{DISCUSSION}

The main result of this study, using two separate multicenter cohorts of critically ill patients with infections, was the consistent association between secretoneurin levels at ICU admission and hospital mortality and shock after adjustment for other 
risk variables in multivariate regression analyses. Secretoneurin also improved the risk prediction as assessed by additional statistical indices; thus, secretoneurin seems to be a promising novel biomarker for risk prediction in critically ill patients with infections.

Mortality rates in patients with severe sepsis are $30-40 \%$ (20). Data from different cohorts of patients with severe sepsis have identified multiple-organ failure, refractory shock, and respiratory failure as the main causes of death (20). Acute myocardial infarction and ventricular arrhythmias also contribute to mortality in severe sepsis; thus, myocardial dysfunction is a major contributor to mortality in critically ill patients with infections as also demonstrated by echocardiography (21-23). Pertinent to this, there is evidence that myocardial dysfunction in severe sepsis may be at least partly preventable, including experimental and retrospective studies that have found positive effects on mortality by $\beta$-blockers and statins (24-26). A prospective single-center study also reported better outcomes in septic shock patients who were randomized to $\beta$-blocker infusion from 24 hours after ICU admission (27); however, 28-day mortality was still $49 \%$ in the $\beta$-blocker group. Accordingly, a biomarker that at ICU admission could identify patients with infections at increased risk of hospital mortality and shock could prove helpful to help optimize the benefit-harm ratio of cardioprotective therapy.

To have clinical utility, a biomarker needs to add to established risk indices and to improve patient management (28). Our data support secretoneurin as a prognostic biomarker in critically ill patients with infections as admission secretoneurin levels provided incremental prognostic information to established risk indices, including history of CVD and established cardiovascular biomarkers. Secretoneurin levels over $175 \mathrm{pmol} / \mathrm{L}$ also provided additional prognostic information to SOFA and SAPS II scores in severe sepsis and septic shock, and this was the case although secretoneurin measurements were performed early after ICU admission, and the risk scores were first calculated after 24 hours. We also found comparable sensitivities and specificities for the cutoff of $175 \mathrm{pmol} / \mathrm{L}$ among septic patients in cohorts \#1 and \#2. Still, the design of this study does not permit any assumptions to be made whether the peptide secretoneurin itself may directly influence outcome in critically ill patients with infections (i.e., causal relationship with outcome) or whether secretoneurin merely is a risk marker that reflects pathophysiology associated with shock and mortality. Our study was neither designed to assess whether secretoneurin measurements may improve patient management; however, patients with high secretoneurin levels, and thus presumably also increased systemic and myocardial stress, could be the patients who benefit the most from cardioprotective therapy. The associations between secretoneurin and neuroendocrine and myocardial dysfunction in our patients are supported by the significant correlations between secretoneurin levels and levels of free cortisol and established cardiovascular biomarkers, the independent association between admission secretoneurin levels and shock in cohort \#1, and the increased need for dobutamine and epinephrine infusion during the ICU stay for patients with high admission secretoneurin levels. Of note, impaired renal function also seems to increase circulating secretoneurin levels, and this could explain part of the prognostic utility of secretoneurin in critically ill patients with infections. Thus, it is possible that secretoneurin may also prove valuable for early detection of renal injury and failure, but this will have to be assessed in additional studies.

The strengths of our study are the use of two separate large multicenter cohorts of critically ill patients with infections and the comparable results in both cohorts. Limitations of the study are the lack of cardiac imaging and no adjudication of cause of death. Cohort \#2 included patients with infections and respiratory failure, but we also validated the cutoff for mortality in severe sepsis and septic shock from cohort \#1 in comparable patients in cohort \#2.

\section{CONCLUSIONS}

Secretoneurin seems to be a biomarker that provides strong and complementary prognostic information to established risk indices in critically ill patients with infections.

\section{ACKNOWLEDGMENTS}

We are grateful for the contribution by all participating centers in the FINNSEPSIS and FINNALI studies. We acknowledge the expert contribution by Inger Olsson, BSc, to the secretoneurin analyses, the contribution by Stepani Bendel, MD, $\mathrm{PhD}$, to the cortisol measurements, and the statistical review by Ms ClaraCecilie Günther, PhD.

\section{REFERENCES}

1. Blanco J, Muriel-Bombín A, Sagredo V, et al; Grupo de Estudios y Análisis en Cuidados Intensivos: Incidence, organ dysfunction and mortality in severe sepsis: A Spanish multicentre study. Crit Care 2008; 12:R158

2. Le Gall JR, Lemeshow S, Saulnier F: A new Simplified Acute Physiology Score (SAPS II) based on a European/North American multicenter study. JAMA 1993; 270:2957-2963

3. Vincent JL, Moreno R, Takala J, et al: The SOFA (Sepsis-related Organ Failure Assessment) score to describe organ dysfunction/failure. On behalf of the Working Group on Sepsis-Related Problems of the European Society of Intensive Care Medicine. Intensive Care Med 1996; 22:707-710

4. Varpula M, Pulkki K, Karlsson S, et al; FINNSEPSIS Study Group: Predictive value of $\mathrm{N}$-terminal pro-brain natriuretic peptide in severe sepsis and septic shock. Crit Care Med 2007; 35:1277-1283

5. Røsjø H, Varpula M, Hagve TA, et al; FINNSEPSIS Study Group: Circulating high sensitivity troponin $\mathrm{T}$ in severe sepsis and septic shock: Distribution, associated factors, and relation to outcome. Intensive Care Med 2011; 37:77-85

6. Bartolomucci A, Possenti R, Mahata SK, et al: The extended granin family: Structure, function, and biomedical implications. Endocr Rev 2011; 32:755-797

7. Røsjø H, Stridsberg M, Florholmen G, et al: Secretogranin II; a protein increased in the myocardium and circulation in heart failure with cardioprotective properties. PLoS One 2012; 7:e37401

8. Røsjø H, Opstad PK, Hoff JE, et al: Effect of short- and long-term physical activities on circulating granin protein levels. Regul Pept 2013; 185:14-19 
9. Ottesen $\mathrm{AH}$, Louch WE, Carlson CR, et al: Secretoneurin is a novel prognostic cardiovascular biomarker associated with cardiomyocyte calcium handling. J Am Coll Cardiol 2015; 65:339-351

10. Hasslacher J, Lehner GF, Harler U, et al: Secretoneurin as a marker for hypoxic brain injury after cardiopulmonary resuscitation. Intensive Care Med 2014; 40:1518-1527

11. Kirchmair R, Gander R, Egger M, et al: The neuropeptide secretoneurin acts as a direct angiogenic cytokine in vitro and in vivo. Circulation $2004 ; 109: 777-783$

12. Albrecht-Schgoer K, Schgoer W, Holfeld J, et al: The angiogenic factor secretoneurin induces coronary angiogenesis in a model of myocardial infarction by stimulation of vascular endothelial growth factor signaling in endothelial cells. Circulation 2012; 126:2491-2501

13. Karlsson S, Varpula M, Ruokonen E, et al: Incidence, treatment, and outcome of severe sepsis in ICU-treated adults in Finland: The Finnsepsis study. Intensive Care Med 2007; 33:435-443

14. Linko R, Okkonen M, Pettilä V, et al; FINNALI-Study Group: Acute respiratory failure in intensive care units. FINNALI: A prospective cohort study. Intensive Care Med 2009; 35:1352-1361

15. Røsjø H, Nygård S, Kaukonen KM, et al; FINNSEPSIS Study Group: Prognostic value of chromogranin A in severe sepsis: Data from the FINNSEPSIS study. Intensive Care Med 2012; 38:820-829

16. Bendel S, Karlsson S, Pettilä V, et al; Finnsepsis Study Group: Free cortisol in sepsis and septic shock. Anesth Analg 2008; 106:1813-1819

17. Cockcroft DW, Gault $\mathrm{MH}$ : Prediction of creatinine clearance from serum creatinine. Nephron 1976; 16:31-41

18. Demler OV, Pencina MJ, D'Agostino RB Sr: Misuse of DeLong test to compare AUCs for nested models. Stat Med 2012; 31:2577-2587
19. Pencina MJ, D'Agostino RB Sr, Steyerberg EW: Extensions of net reclassification improvement calculations to measure usefulness of new biomarkers. Stat Med 2011; 30:11-21

20. Vincent JL, Nelson DR, Williams MD: Is worsening multiple organ failure the cause of death in patients with severe sepsis? Crit Care Med 2011; 39:1050-1055

21. Fernandes CJ Jr, Akamine N, Knobel E: Myocardial depression in sepsis. Shock 2008; 30(Suppl 1):14-17

22. Maeder M, Fehr T, Rickli H, et al: Sepsis-associated myocardial dysfunction: Diagnostic and prognostic impact of cardiac troponins and natriuretic peptides. Chest 2006; 129:1349-1366

23. Landesberg G, Gilon D, Meroz Y, et al: Diastolic dysfunction and mortality in severe sepsis and septic shock. Eur Heart J 2012; 33: 895-903

24. Ackland GL, Yao ST, Rudiger A, et al: Cardioprotection, attenuated systemic inflammation, and survival benefit of beta1-adrenoceptor blockade in severe sepsis in rats. Crit Care Med 2010; 38:388-394

25. Schmidt H, Hennen R, Keller A, et al: Association of statin therapy and increased survival in patients with multiple organ dysfunction syndrome. Intensive Care Med 2006; 32:1248-1251

26. Kruger $\mathrm{P}$, Fitzsimmons $\mathrm{K}$, Cook D, et al: Statin therapy is associated with fewer deaths in patients with bacteraemia. Intensive Care Med 2006; 32:75-79

27. Morelli A, Ertmer C, Westphal M, et al: Effect of heart rate control with esmolol on hemodynamic and clinical outcomes in patients with septic shock: A randomized clinical trial. JAMA 2013; 310:1683-1691

28. Morrow DA, de Lemos JA: Benchmarks for the assessment of novel cardiovascular biomarkers. Circulation 2007; 115:949-952 